\title{
Efficacy of a tiered therapy defibrillator system used to treat recurrent ventricular arrhythmias refractory to drugs
}

Andrew C Rankin, Sina Zaim, Anne Powell, Bülent Zaim, Ross Brooks, Brian A McGovern, Hasan Garan, Jeremy N Ruskin

\begin{abstract}
Objective-To evaluate an implantable tiered therapy defibrillator system that delivered antitachycardia pacing treatment for slower well tolerated ventricular tachycardias and cardioversion or defibrillation for fast tachycardias or ventricular fibrillation.
\end{abstract}

Methods-A tiered treatment device (Ventritex Cadence V-100) was implanted in 30 patients with ventricular tachycardia that was refractory to drugs. Efficacy was evaluated by the responses of induced or spontaneous arrhythmias to the treatments delivered.

Results-Antitachycardia pacing successfully terminated $80 \%$ of episodes of ventricular tachycardia induced by noninvasive programmed stimulation, but acceleration was brought about by pacing in six patients in $10 \%$ of episodes. During a follow up of two to 17 (mean seven) months, 18 patients $(60 \%)$ had recurrence of ventricular arrhythmias. Antitachycardia pacing terminated ventricular tachycardia in 17 of 18 patients in $87 \%$ of episodes. Twelve patients received shocks for ventricular tachycardia or fibrillation. Failure of pacing, with subsequent cardioversion, occurred in nine patients $(50 \%)$ in one or more episodes. Acceleration of tachycardia by pacing occurred in 10 patients in $5 \%$ of episodes. Only two of these patients had experienced acceleration of previously induced arrhythmia. Five patients had spontaneous fast ventricular tachycardia or fibrillation treated by cardioversion or defibrillation. Spurious treatment was delivered in nine patients $(30 \%)$, during atrial fibrillation in five, sinus tachycardia in two, and because of fracture of the sensing lead system in two patients. The retrieval of stored intracardiac electrograms was of clinical value in assessing spurious treatment.

Conclusions-Tiered treatment was effective in terminating recurrent ventricular arrhythmias in these selected patients. Most episodes were treated successfully by pacing, and resistant tachycardias, pacing induced acceleration, or haemodynamically compromising arrhythmias were treated by shocks.

(Br Heart f 1993;70:61-69)

Patients with sustained ventricular arrhyth- mias who have inducible ventricular tachycardia at electrophysiological study despite antiarrhythmic drug treatment are at high risk of recurrence of arrhythmia. ${ }^{12}$ Automatic implantable devices capable of detecting arrhythmias and treating them with cardioversion or defibrillation have greatly diminished the incidence of sudden death in patients with haemodynamically compromising ventricular arrhythmias. ${ }^{34}$ Patients with slower and better tolerated ventricular tachycardia, however, are less well suited for such devices. Their risk of sudden death may be lower, ${ }^{56}$ and they might be subjected to frequent painful shocks with minimal preceding symptoms. It is well established that short bursts of rapid ventricular pacing effectively terminated sustained monomorphic ventricular tachycardia in most patients, but may also unpredictably cause acceleration of the tachycardia. ${ }^{78}$ This potential for tachycardia acceleration has limited the application of antitachycardia pacing in patients with recurrent sustained ventricular tachycardia. ${ }^{9}$ Provision of additional cardioversion and defibrillation capability allows the benefit of pacing termination in most episodes of ventricular tachycardia, without the risks associated with acceleration. Tiered treatment takes this concept further and combines the use of antitachycardia pacing for slower, well tolerated ventricular tachycardia with delivery of low energy shocks to cardiovert resistant tachycardia and higher energy shocks to cardiovert or defibrillate faster tachycardias or ventricular fibrillation, whether spontaneous or induced by pacing. In this report we evaluate the efficacy of an implantable tiered treatment defibrillator system in patients with recurrent ventricular arrhythmias refractory to drugs.

\section{Patients and methods}

\section{PATIENTS}

Thirty patients underwent implantation of an automatic tiered treatment defibrillator system (Ventritex Cadence V-100). There were 23 males and seven females, aged 14-78 (mean 50) years. Patients presented with spontaneous ventricular arrhythmias that proved to be refractory to antiarrhythmic drug treatment (including amiodarone in 19 patients), as assessed by suppression of spontaneous or induced arrhythmias. Twenty two patients $(73 \%)$ had coronary artery disease with previous myocardial infarction, four had cardiomyopathy (dilated in two, hypertrophic 
in one, and restrictive in one), two had valvar heart disease with previous myocardial infarction (one embolic, one perioperative infarction), one had right ventricular dysplasia, and one had idiopathic ventricular fibrillation. The mean (SD) left ventricular ejection fraction was $36 \%(15 \%)$.

\section{SELECTION OF PATIENTS}

Patients were selected for implantation of a tiered treatment device for control of arrhythmias on the basis of combinations of the arrhythmia characteristics: (a) haemodynamically tolerated ventricular tachycardia (spontaneous or induced), (b) recurrent ventricular tachycardia despite antiarrhythmic drug treatment, (c) inducible ventricular tachycardia that was slowed, but not suppressed, by antiarrhythmic drugs, and (d) successful termination of tachycardia by ventricular burst pacing. One patient without inducible sustained arrhythmia was selected to receive the device because of its diagnostic capabilities.

\section{ELECTROPHYSIOLOGICAL TESTING}

Induction of arrhythmias by programmed ventricular stimulation was undertaken at baseline and during drug treatment with up to three ventricular extrastimuli at two basic drive cycle lengths, at one or two right ventricular sites. Attempts were made to terminate induced ventricular tachycardia in all patients with short bursts of rapid right ventricular pacing. Normal rhythm was restored by direct current cardioversion if the tachycardia accelerated with pacing or was not tolerated haemodynamically. Serial electrophysiological testing was performed in all patients undergoing oral antiarrhythmic treatment.

\section{CHARACTERISTICS OF THE DEVICE}

The Ventritex Cadence tiered treatment device has multiple programmable arrhythmia detection criteria and therapeutic options. Detection of arrhythmia is based on the satisfaction of programmed rate criteria with recognition of a tachycardia occurring whenever the sensed rate exceeds the specified detection rate. Also, the device was programmed to discriminate among up to three distinct tachycardia types, based on rate zones (tachycardias A, B, and fibrillation). Programmed detection rates ranged from 120-160 (mean 138) beats/min for the detection of slow ventricular tachycardia (tachycardia A), and from 188 to 222 beats/min for ventricular fibrillation, with intermediate rates for tachycardia $\mathbf{B}$. The treatment delivered was independently programmed for each arrhythmia category. Adaptive decremental ventricular burst pacing was used as initial treatment for tachycardia A. This modality delivered trains of ventricular stimuli at a preprogrammed percentage of the detected tachycardia cycle length, with subsequent shortening of the paced cycle length for successive bursts. Up to 10 bursts with trains of five to 11 stimuli were used, beginning at $70 \%-85 \%$ of the detected cycle length of the tachycardia and decreasing by $10 \mathrm{~ms}$ with each burst to a minimum duration between stimuli of $200 \mathrm{~ms}$. Persistence of tachycardia after pacing treatment, or detection of a more rapid ventricular rate (tachycardia $B$ or fibrillation), resulted in the synchronised direct current shocks. Delivered shocks were programmed from 50 to $750 \mathrm{~V}$ to provide low energy cardioversion (tachycardia A or B), high energy cardioversion (tachycardia B), or defibrillation.

Diagnostic data were retrieved by interrogation of the implanted device and included the total shock counts, a detection and treatment summary (with minimum and maximum cycle lengths of tachycardia), details of treatment sequencing (of the last 11 events), and retrieval of stored intracardiac electrograms $^{10}$ corresponding to the last three detected arrhythmias. Additional features of the device include backup bradycardia pacing, reconfirmation of tachycardia immediately before delivery of treatment, and non invasive programmed stimulation.

\section{IMPLANTATION OF THE DEVICE}

Patients underwent surgical implantation of extrapericardial patch electrodes through a subxiphoid incision (one), median sternotomy (one), or left lateral thoracotomy (28). Sixteen patients had two large patches, and 14 had one large and one small patch. In 17 patients, a transvenous bipolar electrode for sensing and pacing was inserted through the left subclavian vein, with the tip positioned at the right ventricular apex and the lead tunnelled to the generator in an abdominal wall pocket. The remaining 13 patients received unipolar epicardial sutureless sensing-pacing electrodes, connected to the generator through a bifurcating adaptor. Defibrillation thresholds of $20 \mathrm{~J}$ or less (mean $10.7 \mathrm{~J}$ were found in all patients at implantation. Sensing $R$ wave amplitudes were greater than $5 \mathrm{mV}$ (6-20, mean $13 \cdot 7 \mathrm{mV}$ ). High voltage lead impedance ranged from 28 to 55 (mean 40) ohms.

\section{NON-INVASIVE PROGRAMMED STIMULATION}

Non-invasive programmed stimulation was performed by programming the device to deliver trains of ventricular stimuli followed by up to three extrastimuli, or rapid burst pacing. After implantation, with the patient under general anaesthesia, sustained ventricular tachycardia or fibrillation was induced one to five times and was ended by the device. Alterations in the detection criteria or treatment algorithms were made as indicated by the responses of the device to the detected arrhythmias. Patients were followed up as outpatients at four to six weeks and every two months thereafter. Non-invasive programmed stimulation was repeated at the first return visit, at one year, and at other intervals if reprogramming of the device was necessary.

STATISTICAL ANALYSIS

Data are expressed as mean (SD). Comparisons were made with paired $t$ tests 
(for continuous data) or $\chi^{2}$ tests (for categorical data).

\section{Results}

CHARACTERISTICS OF CLINICAL ARRHYTHMIA

All 30 patients had a history of spontaneous ventricular tachycardias (range one to 13 episodes). Sustained monomorphic ventricular tachycardia occurred in 27 patients, ventricular fibrillation in two, and non-sustained ventricular tachycardia with syncope in one patient. Two or more episodes of spontaneous ventricular tachycardia were documented in 25 patients. Twenty patients had a four month to 10 year history of recurrent spontaneous ventricular tachycardia despite antiarrhythmic drug treatment. In these 20 patients, the mean tachycardia cycle length was increased by chronic drug treatment ( 320 $\mathrm{ms} v 413 \mathrm{~ms} ; \mathrm{p}<0.05)$. The remaining 10 patients underwent implantation of the device within one or two months of their first spontaneous ventricular tachycardia.

\section{ELECTROPHYSIOLOGICAL TESTS}

Programmed ventricular stimulation was performed in the drug free state in 26 of the 30 patients. The other four required antiarrhythmic drugs to control spontaneous arrhythmias. Sustained monomorphic ventricular tachycardia was induced in 24 of the 26 patients, and non-sustained ventricular tachycardia was induced in the remaining two patients. The cycle length of the induced tachycardias ranged from 250 to $520 \mathrm{~ms}$ (mean $313 \mathrm{~ms}$ ). Induced sustained ventricular tachycardia was terminated by ventricular burst pacing in 19 patients, but nine patients required direct current cardioversion to end at least one arrhythmia.

Twenty eight of the 30 patients underwent electrophysiological tests during oral drug treatment. Two patients proceded to device implantation without a repeat study because of recurring spontaneous arrhythmia despite antiarrhythmic drugs in one and drug induced heart block in the other. One patient who had inducible non-sustained ventricular tachycardia at baseline study had no inducible arrhythmia at follow up study. The remaining 27 patients did not respond to a mean of three (range one to five) drugs during serial antiarrhythmic drug tests. The rates of induced ventricular tachycardia were slowed by drug treatment (mean cycle length $427 \mathrm{~ms} ; \mathrm{p}<0.0001$ ). Rapid ventricular pacing successfully ended ventricular tachycardia in 25 patients during antiarrhythmic drug treatment, although eight required cardioversion on at least one occasion. The two patients in whom pacing failed to end the ventricular tachycardia during drug treatment both had their arrhythmia terminated successfully by ventricular pacing at the baseline study.

\section{NON-INVASIVE PROGRAMMED STIMULATION}

All patients had non-invasive programmed ventricular stimulation after implantation of the device, before discharge from hospital (fig 1). Arrhythmia was induced once in 11 patients, and twice or more in 18 patients. Antitachycardia pacing terminated 31 of 44 of episodes $(70 \%)$ of induced ventricular tachycardia, with success in 17 patients, failure in eight patients, and acceleration of ventricular tachycardia in four patients (fig 2). Rapid tachycardia, which was faster than the rate for antitachycardia pacing, or ventricular fibrillation were induced at least once in 16 patients and were successfully terminated by cardioversion or defibrillation.

All patients underwent further non-invasive programmed ventricular stimulation during the follow up of two to 17 (mean seven)
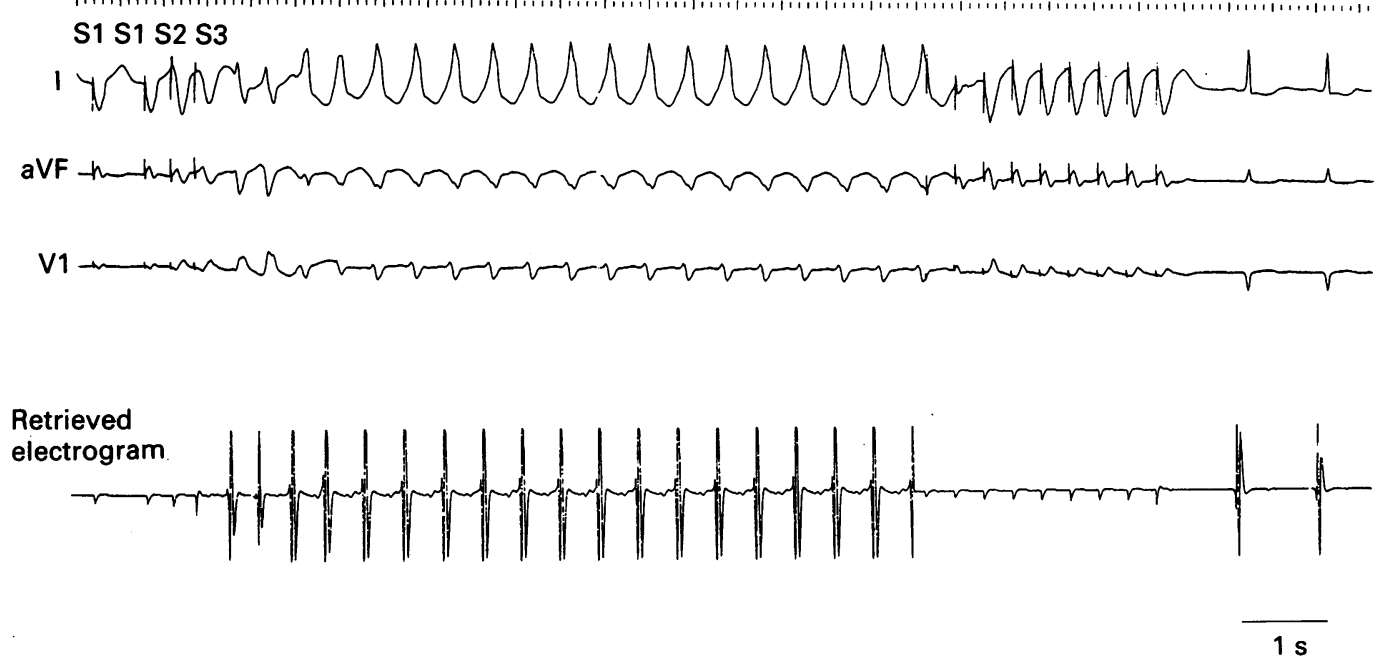

Figure 1 Sustained monomorphic ventricular tachycardia induced by non-invasive programmed stimulation and terminated by antitachycardia pacing. The top traces are surface electrocardiogram leads $(I, a V F, V 1)$ that show the last two paced beats of a drive train of eight beats (S1) followed by two ventricular extrastimuli $(S 2, S 3)$. The induced ventricular tachycardia was detected by the implanted device and terminated by a burst of pacing. The lower trace shows ventricular tachycardia electrograms for this same episode, which were subsequently retrieved from the device. The pacing artefacts appear as low amplitude signals during programmed stimulation and overdrive pacing. The larger signals are artefacts appear as low amplitude signals during programmed stimulation and overdrive pacing. The larger signal 


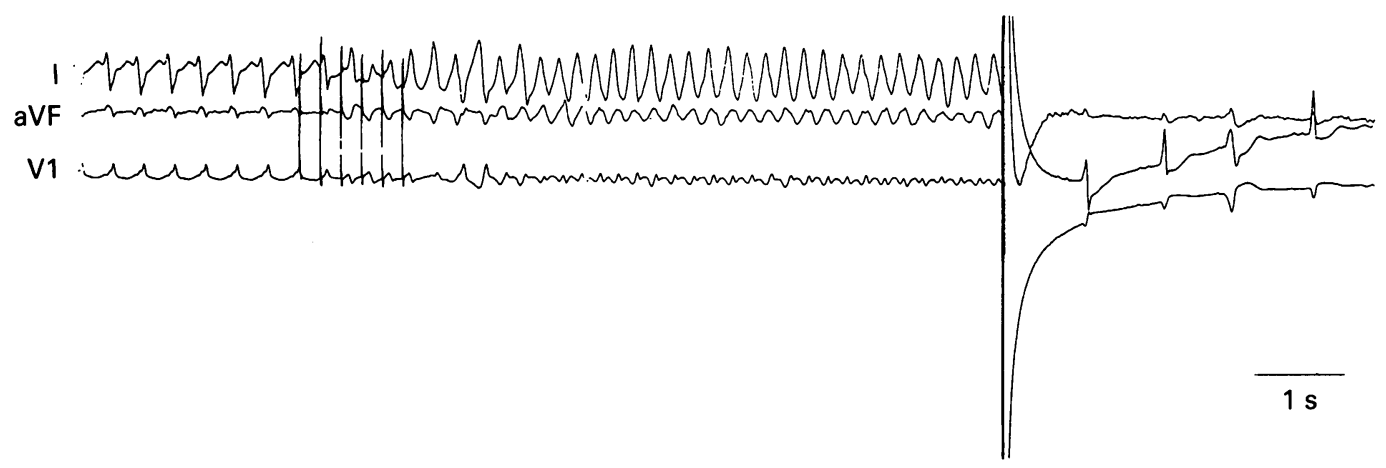

Figure 2 A burst of ventricular pacing, delivered as antitachycardia treatment during sustained monomorphic ventricular tachycardia, caused acceleration to a rapid polymorphic ventricular tachycardia. This tachycardia was faster than the programmed rate zone for antitachycardia pacing, and therefore its detection by the device resulted in the delivery of a 200 $V$ shock, which terminated the arrhythmia. Leads I, aVF, and V1 are shown.

months. Non-invasive testing was carried out from one to five times for each patient, depending on the duration of follow up and the clinical need for reprogramming. The success rate of induced tachycardia termination by antitachycardia pacing was higher than at the study before discharge $(87 \% v$ $70 \% ; \mathrm{p}<0.05)$, with a successful termination of 52 episodes of induced ventricular tachycardia, failure in two, and acceleration in six patients. The failure rate was lower $(3 \% v$ $20 \%$ ) but the incidence of acceleration was unchanged ( $9 \% v 10 \%)$.

In total, successful pacing termination of ventricular tachycardia was confirmed during non-invasive programmed stimulation in 83 of 104 episodes $(80 \%)$ of induced ventricular tachycardia in 24 of the 30 patients $(80 \%)$. Acceleration of ventricular tachycardia by pacing occurred in 10 episodes $(10 \%)$ in six patients. In five of six patients in whom termination of ventricular tachycardia was not demonstrated only rapid ventricular tachycardia was induced by non-invasive programmed stimulation.

RECURRENCE OF SPONTANEOUS ARRHYTHMIA During a follow up period of two to 17 (mean seven months), 18 patients $(60 \%)$ had spontaneous arrhythmias, with a total of over 800 documented episodes. The number of episodes ranged from one to 140 for each patient, with 10 patients having more than 10 episodes of recurrent arrhythmia. The time to first recurrence of arrhythmia was three days to four months (median three weeks) and was within one month in 12 patients $(67 \%)$. All 18 patients had recurrent ventricular tachycardia (cycle length 265 to $500 \mathrm{~ms}$ ), and two patients also had spontaneous ventricular fibrillation. Treatment with antiarrhythmic drugs was continued after implantation of the device in 17 patients, and was subsequently restarted in three patients. $\beta$-Adrenergic receptor blocking agents were continued in 17 patients and digoxin in 13.

\section{ANTITACHYCARDIA PACING}

Episodes of spontaneous ventricular tachycardia were successfully ended by burst pacing in 17 of the 18 patients with recurrent arrhythmia (fig 3). In these 17 patients there were 662 episodes of ventricular tachycardia within the rate for antitachycardia pacing and normal rhythm was restored by pacing in 576 episodes (87\%). Pacing was never successful in one patient, who received 123 shocks during 10 months of follow up, and treatment was therefore restricted to low energy cardioversion or defibrillation. Pacing was inconsistent in terminating induced ventricular tachycardia in this patient, with seven of nine episodes requiring cardioversion. Eight other patients also had episodes of tachycardia that failed to respond to pacing, and were terminated by cardioversion. Antitachycardia pacing, however, was successful in 451 of 503 episodes $(90 \%)$ in these eight patients. Pacing

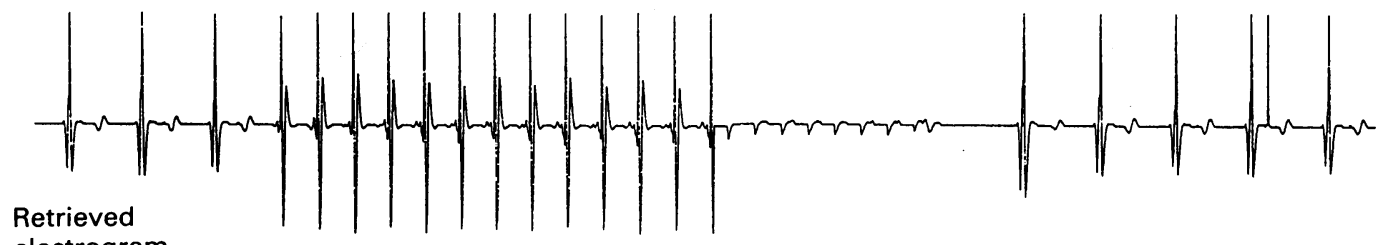

electrogram

Figure 3 Retrieved stored intracardiac electrograms of an episode of spontaneous ventricular tachycardia, detected by the device and terminated by a single burst of antitachycardia pacing. After the third electrogram there was an abrupt increase in ventricular rate that was detected by the implanted tiered treatment device and a burst of antitachycardia pacing was delivered (shown as low amplitude pacing artefacts). During ventricular tachycardia, the intracardiac electrogram morphology was different from that during sinus rhythm, before initiation, and after termination of the tachycardia. The vertical marker after the second to last electrogram was inserted by the device to indicate that it had recognised that the sensed rate has fallen below the tachycardia detection rate. 

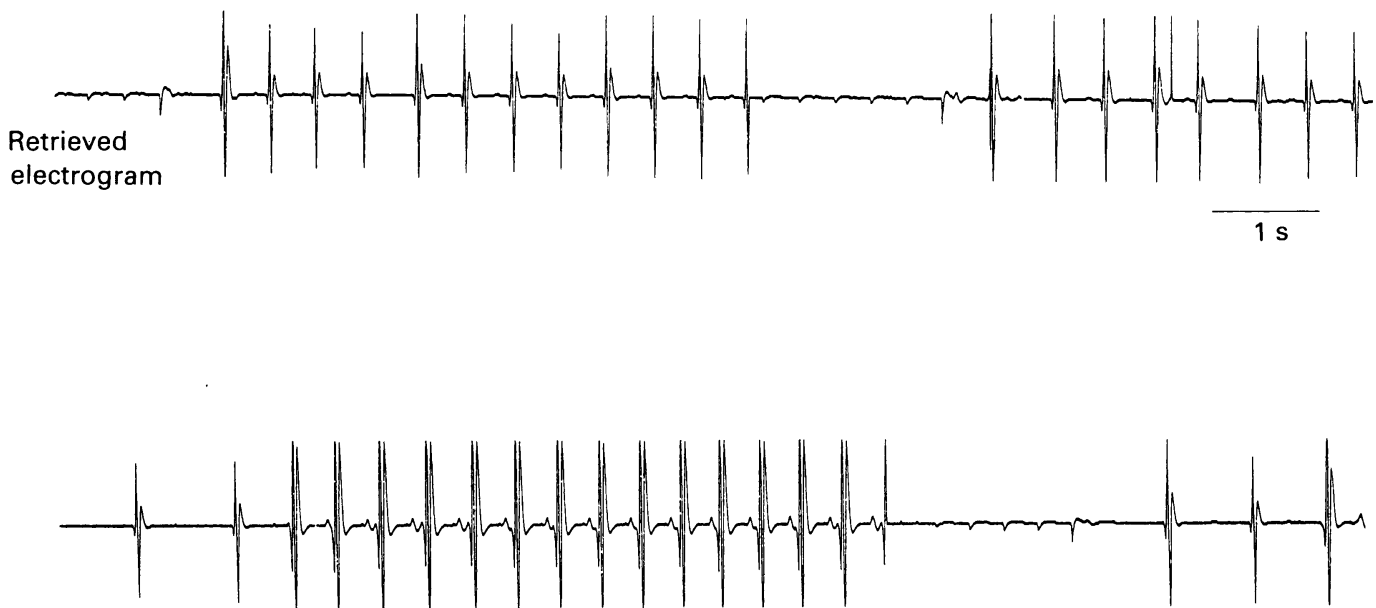

Retrieved electrogram

Figure 4 Antitachycardia pacing during atrial and ventricular arrhythmias. Top trace shows retrieved intracardiac electrograms with pacing during an irregular tachycardia. After the second burst of pacing the vertical marker indicated that the rate had fallen below the tachycardia detection rate, although the tachycardia was not terminated. The electrograms did not change and were of identical morphology to those during sinus rhythm, consistent with an atrial arrhythmia. An episode of ventricular tachycardia in the same patient, successfully terminated by a burst of pacing, is shown below. The electrogram morphology during ventricular tachycardia was different from that during sinus rhythm, before the arrhythmia and after its termination.

had failed to terminate at least one episode of tachycardia induced by non-invasive programmed stimulation in four of these patients.

Acceleration of ventricular tachycardia was documented in 10 patients during 27 of 530 (5\%) paced episodes. The arrhythmia accelerated into the fibrillation rate during 13 episodes in four patients. In only two of the 10 patients had acceleration been found with pacing treatment of arrhythmias induced by non invasive programmed stimulation. Five patients all of whom had infrequent (one to nine) episodes, had all recurrent arrhythmias terminated successfully by pacing.

CARDIOVERSION AND DEFIBRILLATION

Twelve patients received 279 appropriate shocks for ventricular tachycardia or fibrillation (one to 123 shocks/patient). All 12 required cardioversion or defibrillation after antitachycardia pacing, had failed or caused acceleration of ventricular tachycardia. All but one, however, had their arrhythmias successfully terminated on other occasions by pacing. Five patients had spontaneous fast ventricular tachycardia (tachycardia B) or ventricular fibrillation requiring cardioversion or defibrillation; all five had also had episodes of slower tachycardia successfully treated by pacing. The 10 patients with frequently recurring ( $>10$ episodes) arrhythmia all received shocks as well as pacing. Low energy shocks (50-400 V, approximately $0.2-10 \mathrm{~J}$ ) were delivered during ventricular tachycardia in 11 patients, with successful restoration of normal rhythm in eight, failure in four, and acceleration of tachycardia to fibrillation in two patients. Seven patients received high energy shocks (450-750 V, approximately 12-35 J) for termination of ventricular tachycardia or fibrillation. Multiple sequential shocks during a single episode were necessary to terminate arrhythmia in six patients.

SPURIOUS TREATMENT AND UNDERDETECTION The worst problem encountered with programming the device was overlap between the rate of ventricular tachycardia and the rates of sinus tachycardia or atrial arrhythmias, resulting in inappropriate treatment or undersensing of the arrhythmia. Patients with poor left ventricular function or heart failure, who were intolerant of $\beta$-adrenergic receptor blockade, and those whose ventricular tachycardia was slow (often slowed by antiarrhythmic drugs necessary to reduce the frequency of recurrence of arrhythmia) presented the most difficult problem.

Nine patients received treatment from the device for reasons other than ventricular tachycardia, including pacing in four, shocks in two, and both pacing and shocks in three patients. Retrieval of stored intracardiac electrograms was of value in the diagnosis of spurious treatment in these nine patients. Paroxysmal atrial flutter or fibrillation with ventricular response rates within the tachycardia rate detection zone occurred in five patients (fig 4) and sinus tachycardia resulted in the delivery of treatment in two patients with heart failure (fig 5). The retrieved intracardiac electrograms during these tachycardias were of identical morphology to those recorded in sinus rhythm. This was by contrast with the electrograms during spontaneous ventricular tachycardia in the same patients, during which there were changes in both rate and electrogram morphology (fig 4B). Also, the recorded ventricular rhythm was irregular in three patients with atrial fibrillation. The occurrence of atrial flutter, fibrillation, or sinus tachycardia was confirmed by electrocardiographic recordings in 
A

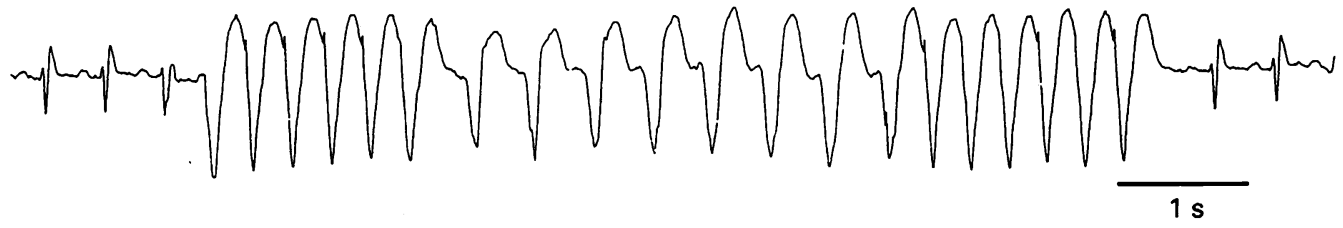

B

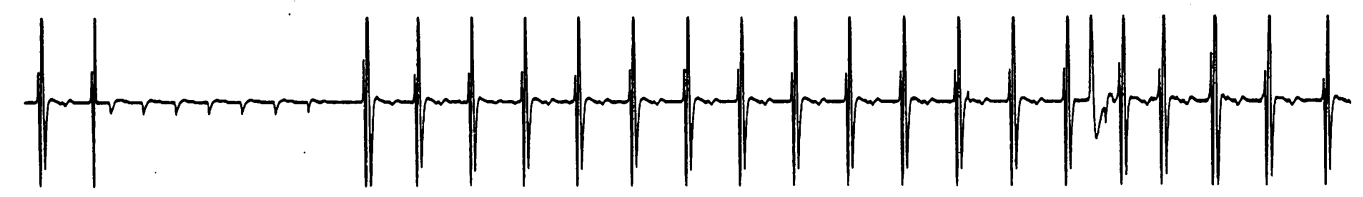

C
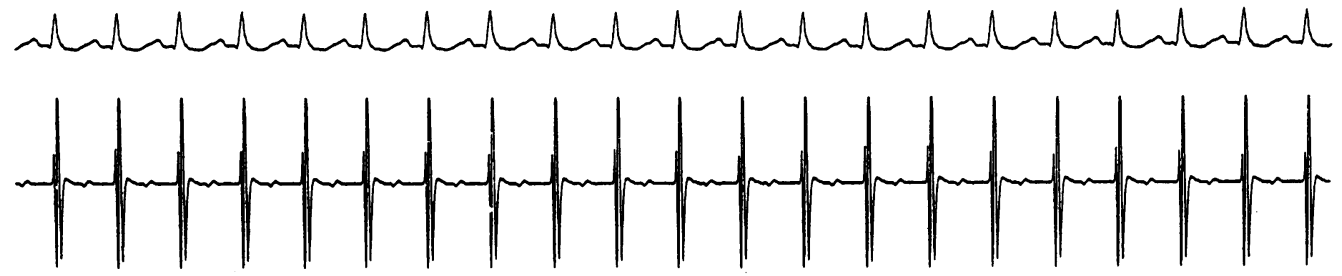

$1 \mathrm{~s}$

Figure 5 Spurious treatment during sinus tachycardia in a patient with severe congestive heart failure. (A) Sinus tachycardia, faster than the programmed arrhythmia detection rate, resulted in a burst of antitachycardia pacing that induced ventricular tachycardia. A second burst of pacing terminated the arrhythmia. The rate of ventricular tachycardia is only slightly faster than that of the sinus tachycardia (surface lead aVF). (B) Retrieved intracardiac electrograms from another episode in the same patient, show pacing treatment and delivery of a shock (arrow) during sinus tachycardia (after failure of pacing to restore normal rate). The electrograms were identical to those recorded during sinus rhythm. (C) Surface lead I and real time intracardiac electrograms recorded during sinus rhythm.

five of the seven patients. A further two patients had multiple shocks, without prior symptoms, and the retrieved electrograms indicated sensed electrical noise (fig 6), sub- sequently found to originate from a fracture in the rate sensing lead system.

Four patients had the converse problem of underdetection of arrhythmia, because of
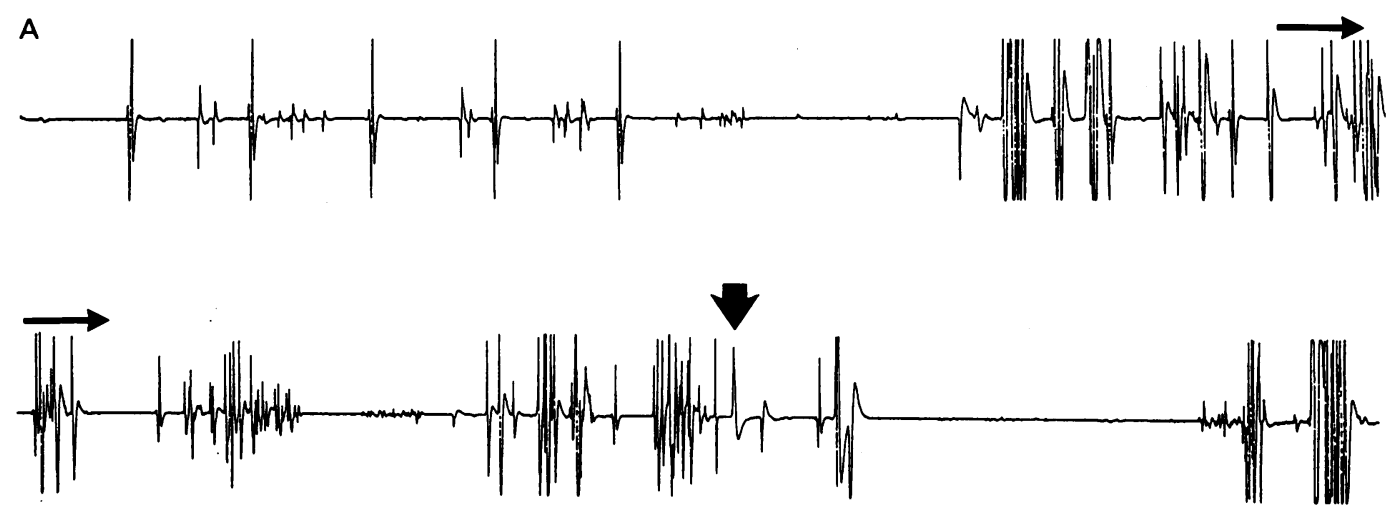

B

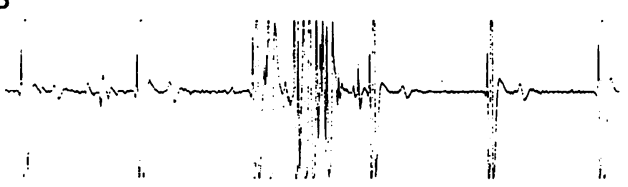

。

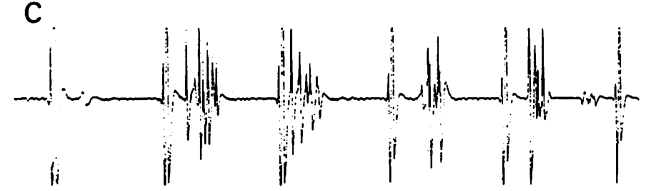

Figure 6 Spurious shock due to noise from sensing lead system fracture. (A) Retrieved stored intracardiac electrograms showing intermittent failure of sensing and high frequency electrical noise that was detected and satisfied the rate criterion for ventricular fibrillation. The device charged, reconfirmed the presence of high frequency activity and delivered a high energy shock (arrow). Similar electrical noise was induced during real time electrogram recordings by the patient bending forward $(B)$ or by moving the device within its abdominal wall pocket $(C)$. A fractured bifurcating lead adaptor was subsequently removed and replaced. 


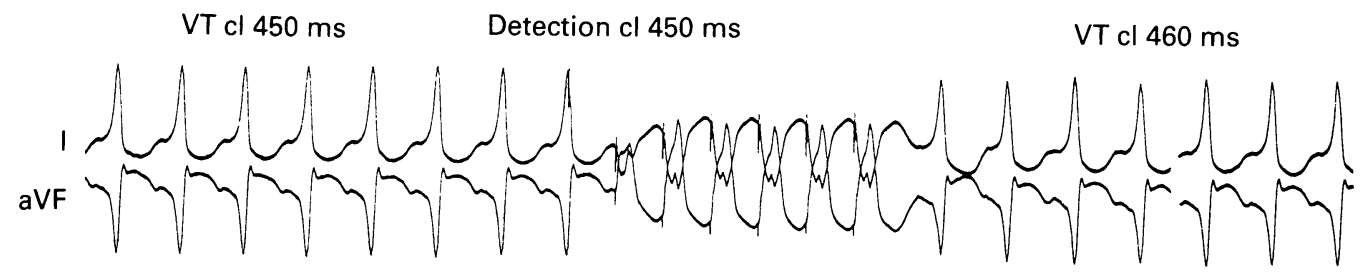

Retrieved electrogram

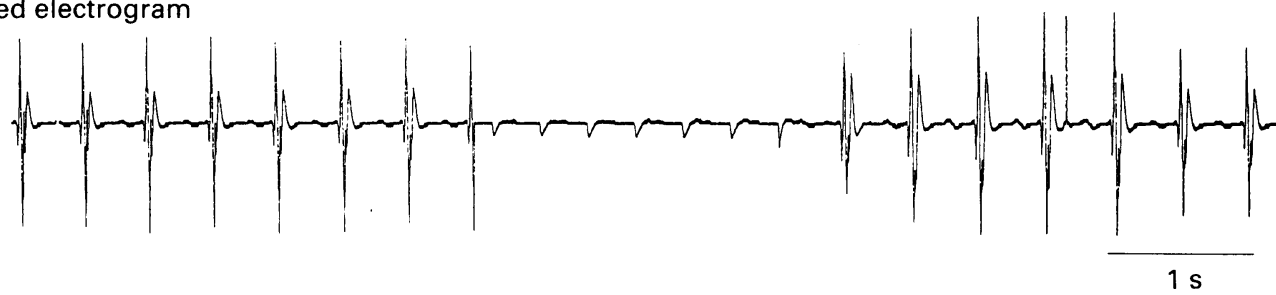

ventricular tachycardia rates that were slower than the programmed detection rates (fig 7). In three of these patients, ventricular tachycardia was initially detected, but transiently fell below the detection rate after pacing treatment (fig 7(A)). Ventricular tachycardia was subsequently redetected and again treated. In these patients the retrieved diagnostic summary of treated arrhythmias was therefore inaccurate as each redetection was categorised as a new event.

CLINICAL COURSE

Twelve patients were admitted to hospital with problems related to the device during their follow up. The most common reason was the delivery of multiple shocks because of recurrent ventricular tachycardia in six patients, atrial fibrillation in two, and fractures of the rate sensing lead system in two patients. One patient had sustained ventricular tachycardia below the programmed arrhythmia detection rate and was admitted to hospital for external cardioversion. The remaining patient had suspected infection of the pulse generator pocket. Three other patients had hospital admissions related to their underlying cardiac disease: congestive heart failure (one patient); chest pain (one patient); and cardiac transplantation (one patient). One patient died from intractable congestive heart failure.

\section{Discussion}

ANTITACHYCARDIA PACING FOR VENTRICULAR TACHYCARDIA

The initiation and termination of sustained monomorphic ventricular tachycardia by premature ventricular extrastimuli first indicated that reentry would be the likely underlying mechanism of the tachycardia. ${ }^{1213}$ Further evidence has supported a reentrant basis for most ventricular tachycardias induced by programmed ventricular stimulation ${ }^{14-16}$ and confirmed their susceptibility to termination by ventricular pacing. ${ }^{71416}$ Appropriately timed single ventricular extrastimuli may terminate a minority (9\%-27\%) induced ventricular tachycardias, but the success rate increases as more extrastimuli are delivered, rising to $36 \%-63 \%$ with double extrastimuli and $48 \%$ $89 \%$ with short bursts of rapid ventricular pacing. ${ }^{781718}$ The success rate is also greater with slower tachycardias, ${ }^{818-22}$ the increase in the diastolic interval allowing a wider excitable gap. Naccarelli et al reported that pacing was successful in $84 \%$ of tachycardias with cycle lengths equal to or greater than $350 \mathrm{~ms}$, compared with $51 \%$ of faster tachycardias. ${ }^{18}$ 
Holley and co-authors reported that cardioversion was invariably required to terminate tachycardias with cycle lengths shorter than $230 \mathrm{~ms}$, but burst pacing was successful in $85 \%$ of slower tachycardias. ${ }^{21}$ Thus in many patients, antiarrhythmic drugs that slow the rate of tachycardia may improve haemodynamic tolerance of the arrhythmia and increase the success rate of antitachycardia pacing. ${ }^{818-20}$ In up to $25 \%$ of patients, however, antiarrhythmic drugs may make tachycardias more difficult to terminate, despite slowing their rate. ${ }^{818}$

\section{ACCELERATION BY PACING OF VENTRICULAR \\ TACHYCARDIA}

The aggressiveness of the pacing protocol not only influences the efficacy of pace termination, but also the risk of pacing induced acceleration of ventricular tachycardia. ${ }^{8}$ The incidence of acceleration increases from $0-0.5 \%$ with single extrastimuli to $15 \%-37 \%$ with burst pacing. ${ }^{81822}$ Whereas pacing induced acceleration of supraventricular arrhythmias may be well tolerated, ${ }^{23}{ }^{24}$ such modification of ventricular tachycardia may be fatal. ${ }^{24} 25$ The use of implantable antitachycardia pacemakers for the treatment of ventricular arrhythmias has, therefore, been limited. ${ }^{24} 25$ Manual activation of antitachycardia pacemakers, by patients or physician, and a few automatic devices, have been used with success in highly selected patients with recurrent monomorphic ventricular tachycardia. ${ }^{24-27}$ Despite rigorous selection criteria, including more than 100 examples of termination of tachycardia without acceleration in each patient, ${ }^{24}$ there have been reports of sudden deaths. ${ }^{23} 24$ The concomitant implantation of a separate automatic cardioverter defibrillator has been used in some patients to overcome this unpredictable risk of pacing induced acceleration of ventricular tachycardia. ${ }^{11}$ 28-30

\section{TIERED TREATMENT}

The third generation antitachycardia pacemaker cardioverter defibrillator in our report not only combines antitachycardia pacing, cardioversion, and defibrillation, but expands substantially the diagnostic and therapeutic options available in an implantable device to control arrhythmia. The device discriminates between rapid ventricular tachycardia, that is likely to be poorly tolerated and has a low probability of successful pace termination, and slower tachycardias for which pacing attempts would be appropriate. The therapeutic options are expanded by the delivery of programmable energy, because organised monomorphic ventricular tachycardia can be cardioverted with considerably lower energies than those required for defibrillation, ${ }^{31}$ with the advantages of less painful shocks and battery conservation.

The present report describes the successful application of tiered electrical treatment in selected patients with recurrent life threatening ventricular arrhythmias. Most episodes of ventricular tachycardia were successfully terminated by pacing, with the advantage of prompt, virtually symptom free treatment. Episodes of tachycardia that were resistant to pacing termination were mainly cardioverted by low energy shocks, and acceleration to fast ventricular tachycardia or fibrillation was treated with high energy shocks. Patients with relatively slow tachycardias may also have episodes of fast tachycardia or fibrillation ${ }^{6}$ and tiered treatment allowed for effective treatment of such episodes. The capability for antitachycardia pacing greatly reduced the number of defibrillator shocks for ventricular tachycardia in these patients ${ }^{32}$ and also made treatment by the device available to some patients with frequent, drug resistant, haemodynamically tolerated ventricular tachycardia who had previously not been considered suitable for treatment by an implantable device.

\section{LIMITATIONS OF TIERED TREATMENT}

The main limitations of tiered devices related not to the function of the device but, rather, to the clinical characteristics of the highly selected patients included in this study. The detection rate criteria necessary to ensure reliable detection of ventricular tachycardia in these patients with slow arrhythmias increased the possibility of spurious pacing treatment during sinus tachycardia or atrial arrhythmias. This was a particular problem in patients with congestive heart failure or intolerance of $\beta$-adrenergic receptor blocking agents, which prevented pharmacological control of heart rate and allowed little margin between sinus and ventricular rates of tachycardia. The $30 \%$ incidence of spurious treatment found in this study was similar to that reported with conventional implantable cardioverter defibrillators in patients with faster arrhythmias. ${ }^{43-35}$ Reconfirmation of persistence of arrhythmia before delivery of treatment may have prevented pacing and shocks for non-sustained arrhythmias. This accounts for a significant proportion of the spurious shocks found with committed defibrillator devices. $^{3433}$

\section{DIAGNOSTIC POTENTIAL}

The diagnostic capabilities of this device were of substantial clinical value, particularly in the diagnosis of spontaneous arrhythmias. It has been recognised with conventional implantable cardioverter defibrillators that the absence of symptoms before the device discharges is not specific for spurious treatment, and may indicate prompt detection and treatment of ventricular arrhythmias, rather than inappropriate treatment. ${ }^{34}$ The diagnosis of spurious treatment is further hindered when the treatment itself may be symptom free, as is often true with antitachycardia pacing. The ability to review the intracardiac electrograms from detected events allowed the objective diagnosis of spurious treatment for sinus tachycardia or supraventricular arrhythmias as well as the prompt detection of sensing lead malfunction. The availability of these advanced diagnostic capabilities will 
aid not only in the management of individual patients ${ }^{10}$ but will contribute importantly to the understanding of the natural history of recurrent ventricular arrhythmias.

\section{RESPONSES TO INDUCED ARRHYTHMIA}

The value of non-invasive programmed stimulation was evident in the lower failure rate of pacing treatment during follow up, compared with the initial responses, before discharge because of improved programming of the antitachycardia pacing options. It is of interest, however, that the incidence of pacing induced acceleration of ventricular tachycardia was not altered during follow up. It is also of note that acceleration by pacing of spontaneous ventricular tachycardia occurred in patients in whom acceleration of induced tachycardia had not occurred during testing of the device in hospital. This may be a result of differences in rates of ventricular tachycardia and autonomic tone during spontaneous episodes of tachycardia. Failure to find pacing induced acceleration in some patients, however, may also have been a function of the fact that only a few arrhythmias were induced by non invasive programmed stimulation before discharge from hospital. Non-invasive programmed stimulation was further limited by the inability to induce the clinical tachycardia in some patients, preventing testing of antitachycardia pacing.

Tiered electrical treatment was safe and highly effective for selected patients with recurrent drug resistant ventricular arrhythmias. The availability of detection of many categories of arrhythmia, antitachycardia pacing modes combined with low energy cardioversion, or defibrillation capabilities, and a wide range of advanced diagnostic features constitutes a significant advance in the treatment of patients with life threatening ventricular arrhythmias.

ACR was a British-American Research Fellow of the American Heart Association and British Heart Foundation. His present address is Department of Medical Cardiology, Glasgow Royal Infirmary, Scotland.

1 Swerdlow CD, Winkle RA, Mason JW. Determinants of NEngl f Med 1983;308:1436-42.

2 Wilber DJ, Garan H, Finkelstein D, Kelly E, Newell J, McGovern B, Ruskin JN. Out-of-hospital cardiac arrest. Use of electrophysiologic testing in the prediction of Use of electrophysiologic testing in the prediction
long-term outcome. $N$ Engl $\mathcal{Y} M$ ed 1988;318:19-24.

3 Winkle RA, Mead RH, Ruder MA, et al. Long-term outcome with the automatic implantable cardiovertercome with the automatic implantable cardio

4 Kelly PA, Cannom DS, Garan H, et al. The automatic implantable cardioverter-defibrillator: efficacy, complications, and survival in patients with malignant ventricular arrhythmias. $\mathcal{F}$ Am Coll Cardiol 1988;11:1278-86.

5 Waller TJ, Kay HR, Spielman SR, Kutalek SP, Greensplan AM, Horowitz LN. Reduction in sudden death and total mortality by antiarrhythmic therapy evaluated by electrophysiologic drug testing: criteria of efficacy in patients with sustained ventricular tachyarrhythmia. $\mathcal{F}$ Am Coll Cardiol 1987;10:83-9.

6 Kadish AH, Buxton AE, Waxman HL, Flores B, Josephson ME, Marchlinski FE. Usefulness of electrophysiologic study to determine the clinical tolerance of physiologic study to determine the clinical tolerance of
arrhythmia recurrences during amiodarone therapy. arrhythmia recurrences during

7 Fisher JD, Mehra R, Furman S. Termination of ventricular tachycardia with bursts of rapid ventricular pacing. Am $\mathcal{F}$ Cardiol 1978;41:94-102

8 Roy D, Waxman HL, Buxton AE, et al. Termination of ventricular tachycardia: role of tachycardia cycle length Am $\mathcal{F}$ Cardiol 1982;50:1346-50.

9 Fisher JD, Kim SG, Furman S, Matos JA. Role of implantable pacemakers in control of recurrent ventricular tachy Am $\mathcal{F}$ Cardiol 1982;49:194-206.

10 Hook BG, Marchlinski FE. Value of electrogram recordings in the diagnosis of arrhythmias precipitating electrical device shock therapy. $\mathcal{F}$ Am Coll Cardiol 1991;17 985-90.

11 Greve H, Koch TH, Gulker H, Heuer H. Terminations of malignant ventricular tachycardias by use of an automatic defibrillator (AICD) in combination with an antitachycardia pacemaker. PACE 1988;11:2040-4.

12 Wellens HJ, Schuilenburg RM, Durrer D. Electrical stimulation of the heart in patients with ventricular tachycardia. Circulation 1972;46:216-26.

13 Wellens HJ, Duren DR, Lie KI. Observations on mecha nisms of ventricular tachycardia. Circulation 1976;54 237-44.

14 Almendral JM, Rosenthal ME, Stamato NJ, et al. Analysis of the resetting phenomenon in sustained uniform ventricular tachycardia: incidence and relation to termination. $\mathcal{F}$ Am Coll Cardiol 1986;8:294-300.

14 Gottlieb CD, Rosenthal ME, Stamato NJ, et al. A quantitative evaluation of refractoriness within a reentrant circuit during ventricular tachycardia. Relation to termination. Circulation 1990;82:1289-95.

16 Kay GN, Epstein AE, Plumb VJ. Resetting of ventricular tachycardia by single extrastimuli. Relation to slow conduction within the reentrant circuit. Circulation 1990, 81:507-19.

16 Mason JW, Winkle RA. Electrode-catheter arrhythmia induction in the selection and assessment of antiarrhythmic drug therapy for recurrent ventricular tachycardia. Circulation 1978;58:971-85.

18 Naccarelli GV, Zipes DP, Rahilly GT, Heger J, Prystowski EN. Influence of tachycardia cycle length and antiarrhythmic drugs on pacing termination and acceleration of ventricular tachycardia. Am Heart $\mathcal{f}$ 1983;105:1-5

19 Fisher JD, Kim SG, Matos JA, Ostrow E. Comparative effectiveness of pacing techniques for termination of well-tolerated sustained ventricular tachycardia. $P A C E$ 1983;6:915-22.

20 Kewren G, Miura DS, Somberg JC. Pacing termination of ventricular tachycardia: influence of antiarrhythmicslowed ectopic rate. Am Heart $\mathcal{F}$ 1984;107:638-43.

21 Holley LK, Copper M, Uther JB, Ross DA. Safety and efficacy of pacing for ventricular tachycardia. PACE efficacy of pacing

22 Waldecker B, Brugada P, Zehender M, Stevenson W, den Dulk K, Wellens HJJ. Importance of modes of electrical termination of ventricular tachycardia for the selection termination of ventricular tachycardia for the selection of implantable

23 Waldecker B, Brugada $P$, den Dulk $\mathrm{K}$, Zehender $M$, Wellens HJ. Arrhythmias induced during termination of supraventricular tachycardia. Am $\mathcal{f}$ Cardiol $1985 ; 55$. 412-7.

24 Fisher JD, Johnston DR, Furman S, Mercando AD, Kim SG. Long-term efficacy of antitachycardia pacing for supraventricular and ventricular tachycardias. $A m \mathcal{F}$ Cardiol 1987;60:1311-6.

25 Fisher JD, Johnston DR, Kim SG, Furman S, Mercando AM. Implantable pacers for tachycardia termination: stimulation techniques and long-term efficacy. $P A C E$ 1986;9:1325-33.

26 Ruskin IN, Garan H, Poulin F, Harthorne JW. Permanent radiofrequency ventricular pacing for management of drug-resistant ventricular tachycardia. $\mathrm{Am} \mathcal{F}$ Cardiol 198046:317-21.

27 den Dulk K, Bertholet M, Brugada P, et al. A versatile pacemaker system for termination of tachycardias. $\operatorname{Am} \mathcal{F}$ Cardiol 1983;52:731-8.

28 Manz M, Gerckens U, Funke HD, Kirchhoff PG, Luderitz B. Combination of antitachycardia pacemaker and automatic implantable cardioverter/defibrillator for ventricular tachycardia. PACE 1986;9:676-84

29 Newman DM, Lee MA, Herre JM, Langberg J, Scheinman MM, Griffin JC. Permanent antitachycardia pacemaker therapy for ventricular tachycardia. $P A C E$

30 Epstein AE, Kay GN, Plumb VJ, Shepard RB, Kirklin JK. Combined automatic implantable cardioverter-defibrillator and pacemaker systems: implantation techniques and follow-up. $\mathcal{F} \mathrm{Am}$ Coll Cardiol 1989;13:121-31.

31 Waspe LE Kim SF, Matos JA, Fisher JD. Role of a catheter lead system for transvenous countershock and pacing during electrophysiologic tests: an assessment of the usefulness of catheter shocks for terminating ventricular tachycardia. Am $\mathcal{F}$ Cardiol 1983;52:477-84

32 Leitch JW, Gillis AM, Wyse DG, et al. Reduction in defibrillator shocks with an implantable device combining antitachycardia pacing and shock therapy. $₹ \mathrm{Am}$ Coll Cardiol 1991;18:145-51.

33 Gabry MD, Brodman R, Johnston D, et al. Automatic implantable cardioverter-defibrillator: patient survival, battery longevity, and shock delivery analysis. $7 \mathrm{Am}$ Coll Cardiol 1987;9:1349-56.

34 Fogoros RN, Elson JJ, Bonnet CA. Actuarial incidence and pattern of occurrence of shocks after implantation and pattern of occurrence of shocks after implantation
of the automatic implantable cardioverter defibrillator. of the automatic implan

35 Manolis AS, Tan-DeGuzman W, Lee MA, et al. Clinical experience in seventy-seven patients with the automatic 1989;118:445-50. 